\title{
Reverse Mathematics and Ramsey Properties of Partial Orderings
}

\author{
Jared Corduan and Marcia Groszek
}

\begin{abstract}
A partial ordering $\mathbb{P}$ is $n$-Ramsey if, for every coloring of $n$-element chains from $\mathbb{P}$ in finitely many colors, $\mathbb{P}$ has a homogeneous subordering isomorphic to $\mathbb{P}$. In their paper on Ramsey properties of the complete binary tree, Chubb, Hirst, and McNicholl ask about Ramsey properties of other partial orderings. They also ask whether there is some Ramsey property for pairs equivalent to $A C A_{0}$ over $R C A_{0}$.

A characterization theorem for finite-level partial orderings with Ramsey properties has been proven by the second author. We show, over $R C A_{0}$, that one direction of the equivalence given by this theorem is equivalent to $A C A_{0}$ (for $n \geq 3$ ), and the other is provable in $A T R_{0}$.

We answer Chubb, Hirst, and McNicholl's second question by showing that there is a primitive recursive partial ordering $\mathbb{P}$ such that, over $R C A_{0}$, " $\mathbb{P}$ is 2-Ramsey" is equivalent to $A C A_{0}$.
\end{abstract}

\section{Introduction}

A fruitful branch of inquiry in reverse mathematics has been combinatorics, in particular, Ramsey's theorem [17] and variants thereof.

The infinitary Ramsey theorem for colorings of $n$-tuples, for standard $n \geq 3$, is equivalent to $A C A_{0}$ over $R C A_{0}$ (Simpson [19]). For colorings of 1-tuples, Ramsey's theorem becomes the infinite pigeonhole principle, which was shown by Hirst [11] to be equivalent to $B \Sigma_{2}^{0}$ over $R C A_{0}$. Ramsey's theorem for pairs is a more complicated case. It is strictly weaker than $A C A_{0}$ (Seetpun, see [18] and [12]), and not equivalent to any of the standard second-order systems. Some recent results in the ongoing investigation of the strength of Ramsey's theorem for pairs and of related, generally weaker, combinatorial results, can be found in Cholak, Jockusch, and Slaman [1],

Received June 11, 2012; accepted July 23, 2013

First published online September 9, 2015

2010 Mathematics Subject Classification: Primary 03B30; Secondary 05C55, 03F35

Keywords: reverse mathematics, Ramsey, partitions, partial orderings

(C) 2016 by University of Notre Dame 10.1215/00294527-3314771 


\title{
Reverse Mathematics and Ramsey Properties of Partial Orderings
}

\author{
Jared Corduan and Marcia Groszek
}

\begin{abstract}
A partial ordering $\mathbb{P}$ is $n$-Ramsey if, for every coloring of $n$-element chains from $\mathbb{P}$ in finitely many colors, $\mathbb{P}$ has a homogeneous subordering isomorphic to $\mathbb{P}$. In their paper on Ramsey properties of the complete binary tree, Chubb, Hirst, and McNicholl ask about Ramsey properties of other partial orderings. They also ask whether there is some Ramsey property for pairs equivalent to $A C A_{0}$ over $R C A_{0}$.

A characterization theorem for finite-level partial orderings with Ramsey properties has been proven by the second author. We show, over $R C A_{0}$, that one direction of the equivalence given by this theorem is equivalent to $A C A_{0}$ (for $n \geq 3$ ), and the other is provable in $A T R_{0}$.

We answer Chubb, Hirst, and McNicholl's second question by showing that there is a primitive recursive partial ordering $\mathbb{P}$ such that, over $R C A_{0}$, " $\mathbb{P}$ is 2-Ramsey" is equivalent to $A C A_{0}$.
\end{abstract}

\section{Introduction}

A fruitful branch of inquiry in reverse mathematics has been combinatorics, in particular, Ramsey's theorem [17] and variants thereof.

The infinitary Ramsey theorem for colorings of $n$-tuples, for standard $n \geq 3$, is equivalent to $A C A_{0}$ over $R C A_{0}$ (Simpson [19]). For colorings of 1-tuples, Ramsey's theorem becomes the infinite pigeonhole principle, which was shown by Hirst [11] to be equivalent to $B \Sigma_{2}^{0}$ over $R C A_{0}$. Ramsey's theorem for pairs is a more complicated case. It is strictly weaker than $A C A_{0}$ (Seetpun, see [18] and [12]), and not equivalent to any of the standard second-order systems. Some recent results in the ongoing investigation of the strength of Ramsey's theorem for pairs and of related, generally weaker, combinatorial results, can be found in Cholak, Jockusch, and Slaman [1],

Received June 11, 2012; accepted July 23, 2013

First published online September 9, 2015

2010 Mathematics Subject Classification: Primary 03B30; Secondary 05C55, 03F35

Keywords: reverse mathematics, Ramsey, partitions, partial orderings

(C) 2016 by University of Notre Dame 10.1215/00294527-3314771 


\title{
Reverse Mathematics and Ramsey Properties of Partial Orderings
}

\author{
Jared Corduan and Marcia Groszek
}

\begin{abstract}
A partial ordering $\mathbb{P}$ is $n$-Ramsey if, for every coloring of $n$-element chains from $\mathbb{P}$ in finitely many colors, $\mathbb{P}$ has a homogeneous subordering isomorphic to $\mathbb{P}$. In their paper on Ramsey properties of the complete binary tree, Chubb, Hirst, and McNicholl ask about Ramsey properties of other partial orderings. They also ask whether there is some Ramsey property for pairs equivalent to $A C A_{0}$ over $R C A_{0}$.

A characterization theorem for finite-level partial orderings with Ramsey properties has been proven by the second author. We show, over $R C A_{0}$, that one direction of the equivalence given by this theorem is equivalent to $A C A_{0}$ (for $n \geq 3$ ), and the other is provable in $A T R_{0}$.

We answer Chubb, Hirst, and McNicholl's second question by showing that there is a primitive recursive partial ordering $\mathbb{P}$ such that, over $R C A_{0}$, " $\mathbb{P}$ is 2-Ramsey" is equivalent to $A C A_{0}$.
\end{abstract}

\section{Introduction}

A fruitful branch of inquiry in reverse mathematics has been combinatorics, in particular, Ramsey's theorem [17] and variants thereof.

The infinitary Ramsey theorem for colorings of $n$-tuples, for standard $n \geq 3$, is equivalent to $A C A_{0}$ over $R C A_{0}$ (Simpson [19]). For colorings of 1-tuples, Ramsey's theorem becomes the infinite pigeonhole principle, which was shown by Hirst [11] to be equivalent to $B \Sigma_{2}^{0}$ over $R C A_{0}$. Ramsey's theorem for pairs is a more complicated case. It is strictly weaker than $A C A_{0}$ (Seetpun, see [18] and [12]), and not equivalent to any of the standard second-order systems. Some recent results in the ongoing investigation of the strength of Ramsey's theorem for pairs and of related, generally weaker, combinatorial results, can be found in Cholak, Jockusch, and Slaman [1],

Received June 11, 2012; accepted July 23, 2013

First published online September 9, 2015

2010 Mathematics Subject Classification: Primary 03B30; Secondary 05C55, 03F35

Keywords: reverse mathematics, Ramsey, partitions, partial orderings

(C) 2016 by University of Notre Dame 10.1215/00294527-3314771 


\title{
Reverse Mathematics and Ramsey Properties of Partial Orderings
}

\author{
Jared Corduan and Marcia Groszek
}

\begin{abstract}
A partial ordering $\mathbb{P}$ is $n$-Ramsey if, for every coloring of $n$-element chains from $\mathbb{P}$ in finitely many colors, $\mathbb{P}$ has a homogeneous subordering isomorphic to $\mathbb{P}$. In their paper on Ramsey properties of the complete binary tree, Chubb, Hirst, and McNicholl ask about Ramsey properties of other partial orderings. They also ask whether there is some Ramsey property for pairs equivalent to $A C A_{0}$ over $R C A_{0}$.

A characterization theorem for finite-level partial orderings with Ramsey properties has been proven by the second author. We show, over $R C A_{0}$, that one direction of the equivalence given by this theorem is equivalent to $A C A_{0}$ (for $n \geq 3$ ), and the other is provable in $A T R_{0}$.

We answer Chubb, Hirst, and McNicholl's second question by showing that there is a primitive recursive partial ordering $\mathbb{P}$ such that, over $R C A_{0}$, " $\mathbb{P}$ is 2-Ramsey" is equivalent to $A C A_{0}$.
\end{abstract}

\section{Introduction}

A fruitful branch of inquiry in reverse mathematics has been combinatorics, in particular, Ramsey's theorem [17] and variants thereof.

The infinitary Ramsey theorem for colorings of $n$-tuples, for standard $n \geq 3$, is equivalent to $A C A_{0}$ over $R C A_{0}$ (Simpson [19]). For colorings of 1-tuples, Ramsey's theorem becomes the infinite pigeonhole principle, which was shown by Hirst [11] to be equivalent to $B \Sigma_{2}^{0}$ over $R C A_{0}$. Ramsey's theorem for pairs is a more complicated case. It is strictly weaker than $A C A_{0}$ (Seetpun, see [18] and [12]), and not equivalent to any of the standard second-order systems. Some recent results in the ongoing investigation of the strength of Ramsey's theorem for pairs and of related, generally weaker, combinatorial results, can be found in Cholak, Jockusch, and Slaman [1],

Received June 11, 2012; accepted July 23, 2013

First published online September 9, 2015

2010 Mathematics Subject Classification: Primary 03B30; Secondary 05C55, 03F35

Keywords: reverse mathematics, Ramsey, partitions, partial orderings

(C) 2016 by University of Notre Dame 10.1215/00294527-3314771 


\title{
Reverse Mathematics and Ramsey Properties of Partial Orderings
}

\author{
Jared Corduan and Marcia Groszek
}

\begin{abstract}
A partial ordering $\mathbb{P}$ is $n$-Ramsey if, for every coloring of $n$-element chains from $\mathbb{P}$ in finitely many colors, $\mathbb{P}$ has a homogeneous subordering isomorphic to $\mathbb{P}$. In their paper on Ramsey properties of the complete binary tree, Chubb, Hirst, and McNicholl ask about Ramsey properties of other partial orderings. They also ask whether there is some Ramsey property for pairs equivalent to $A C A_{0}$ over $R C A_{0}$.

A characterization theorem for finite-level partial orderings with Ramsey properties has been proven by the second author. We show, over $R C A_{0}$, that one direction of the equivalence given by this theorem is equivalent to $A C A_{0}$ (for $n \geq 3$ ), and the other is provable in $A T R_{0}$.

We answer Chubb, Hirst, and McNicholl's second question by showing that there is a primitive recursive partial ordering $\mathbb{P}$ such that, over $R C A_{0}$, " $\mathbb{P}$ is 2-Ramsey" is equivalent to $A C A_{0}$.
\end{abstract}

\section{Introduction}

A fruitful branch of inquiry in reverse mathematics has been combinatorics, in particular, Ramsey's theorem [17] and variants thereof.

The infinitary Ramsey theorem for colorings of $n$-tuples, for standard $n \geq 3$, is equivalent to $A C A_{0}$ over $R C A_{0}$ (Simpson [19]). For colorings of 1-tuples, Ramsey's theorem becomes the infinite pigeonhole principle, which was shown by Hirst [11] to be equivalent to $B \Sigma_{2}^{0}$ over $R C A_{0}$. Ramsey's theorem for pairs is a more complicated case. It is strictly weaker than $A C A_{0}$ (Seetpun, see [18] and [12]), and not equivalent to any of the standard second-order systems. Some recent results in the ongoing investigation of the strength of Ramsey's theorem for pairs and of related, generally weaker, combinatorial results, can be found in Cholak, Jockusch, and Slaman [1],

Received June 11, 2012; accepted July 23, 2013

First published online September 9, 2015

2010 Mathematics Subject Classification: Primary 03B30; Secondary 05C55, 03F35

Keywords: reverse mathematics, Ramsey, partitions, partial orderings

(C) 2016 by University of Notre Dame 10.1215/00294527-3314771 


\title{
Reverse Mathematics and Ramsey Properties of Partial Orderings
}

\author{
Jared Corduan and Marcia Groszek
}

\begin{abstract}
A partial ordering $\mathbb{P}$ is $n$-Ramsey if, for every coloring of $n$-element chains from $\mathbb{P}$ in finitely many colors, $\mathbb{P}$ has a homogeneous subordering isomorphic to $\mathbb{P}$. In their paper on Ramsey properties of the complete binary tree, Chubb, Hirst, and McNicholl ask about Ramsey properties of other partial orderings. They also ask whether there is some Ramsey property for pairs equivalent to $A C A_{0}$ over $R C A_{0}$.

A characterization theorem for finite-level partial orderings with Ramsey properties has been proven by the second author. We show, over $R C A_{0}$, that one direction of the equivalence given by this theorem is equivalent to $A C A_{0}$ (for $n \geq 3$ ), and the other is provable in $A T R_{0}$.

We answer Chubb, Hirst, and McNicholl's second question by showing that there is a primitive recursive partial ordering $\mathbb{P}$ such that, over $R C A_{0}$, " $\mathbb{P}$ is 2-Ramsey" is equivalent to $A C A_{0}$.
\end{abstract}

\section{Introduction}

A fruitful branch of inquiry in reverse mathematics has been combinatorics, in particular, Ramsey's theorem [17] and variants thereof.

The infinitary Ramsey theorem for colorings of $n$-tuples, for standard $n \geq 3$, is equivalent to $A C A_{0}$ over $R C A_{0}$ (Simpson [19]). For colorings of 1-tuples, Ramsey's theorem becomes the infinite pigeonhole principle, which was shown by Hirst [11] to be equivalent to $B \Sigma_{2}^{0}$ over $R C A_{0}$. Ramsey's theorem for pairs is a more complicated case. It is strictly weaker than $A C A_{0}$ (Seetpun, see [18] and [12]), and not equivalent to any of the standard second-order systems. Some recent results in the ongoing investigation of the strength of Ramsey's theorem for pairs and of related, generally weaker, combinatorial results, can be found in Cholak, Jockusch, and Slaman [1],

Received June 11, 2012; accepted July 23, 2013

First published online September 9, 2015

2010 Mathematics Subject Classification: Primary 03B30; Secondary 05C55, 03F35

Keywords: reverse mathematics, Ramsey, partitions, partial orderings

(C) 2016 by University of Notre Dame 10.1215/00294527-3314771 


\title{
Reverse Mathematics and Ramsey Properties of Partial Orderings
}

\author{
Jared Corduan and Marcia Groszek
}

\begin{abstract}
A partial ordering $\mathbb{P}$ is $n$-Ramsey if, for every coloring of $n$-element chains from $\mathbb{P}$ in finitely many colors, $\mathbb{P}$ has a homogeneous subordering isomorphic to $\mathbb{P}$. In their paper on Ramsey properties of the complete binary tree, Chubb, Hirst, and McNicholl ask about Ramsey properties of other partial orderings. They also ask whether there is some Ramsey property for pairs equivalent to $A C A_{0}$ over $R C A_{0}$.

A characterization theorem for finite-level partial orderings with Ramsey properties has been proven by the second author. We show, over $R C A_{0}$, that one direction of the equivalence given by this theorem is equivalent to $A C A_{0}$ (for $n \geq 3$ ), and the other is provable in $A T R_{0}$.

We answer Chubb, Hirst, and McNicholl's second question by showing that there is a primitive recursive partial ordering $\mathbb{P}$ such that, over $R C A_{0}$, " $\mathbb{P}$ is 2-Ramsey" is equivalent to $A C A_{0}$.
\end{abstract}

\section{Introduction}

A fruitful branch of inquiry in reverse mathematics has been combinatorics, in particular, Ramsey's theorem [17] and variants thereof.

The infinitary Ramsey theorem for colorings of $n$-tuples, for standard $n \geq 3$, is equivalent to $A C A_{0}$ over $R C A_{0}$ (Simpson [19]). For colorings of 1-tuples, Ramsey's theorem becomes the infinite pigeonhole principle, which was shown by Hirst [11] to be equivalent to $B \Sigma_{2}^{0}$ over $R C A_{0}$. Ramsey's theorem for pairs is a more complicated case. It is strictly weaker than $A C A_{0}$ (Seetpun, see [18] and [12]), and not equivalent to any of the standard second-order systems. Some recent results in the ongoing investigation of the strength of Ramsey's theorem for pairs and of related, generally weaker, combinatorial results, can be found in Cholak, Jockusch, and Slaman [1],

Received June 11, 2012; accepted July 23, 2013

First published online September 9, 2015

2010 Mathematics Subject Classification: Primary 03B30; Secondary 05C55, 03F35

Keywords: reverse mathematics, Ramsey, partitions, partial orderings

(C) 2016 by University of Notre Dame 10.1215/00294527-3314771 


\title{
Reverse Mathematics and Ramsey Properties of Partial Orderings
}

\author{
Jared Corduan and Marcia Groszek
}

\begin{abstract}
A partial ordering $\mathbb{P}$ is $n$-Ramsey if, for every coloring of $n$-element chains from $\mathbb{P}$ in finitely many colors, $\mathbb{P}$ has a homogeneous subordering isomorphic to $\mathbb{P}$. In their paper on Ramsey properties of the complete binary tree, Chubb, Hirst, and McNicholl ask about Ramsey properties of other partial orderings. They also ask whether there is some Ramsey property for pairs equivalent to $A C A_{0}$ over $R C A_{0}$.

A characterization theorem for finite-level partial orderings with Ramsey properties has been proven by the second author. We show, over $R C A_{0}$, that one direction of the equivalence given by this theorem is equivalent to $A C A_{0}$ (for $n \geq 3$ ), and the other is provable in $A T R_{0}$.

We answer Chubb, Hirst, and McNicholl's second question by showing that there is a primitive recursive partial ordering $\mathbb{P}$ such that, over $R C A_{0}$, " $\mathbb{P}$ is 2-Ramsey" is equivalent to $A C A_{0}$.
\end{abstract}

\section{Introduction}

A fruitful branch of inquiry in reverse mathematics has been combinatorics, in particular, Ramsey's theorem [17] and variants thereof.

The infinitary Ramsey theorem for colorings of $n$-tuples, for standard $n \geq 3$, is equivalent to $A C A_{0}$ over $R C A_{0}$ (Simpson [19]). For colorings of 1-tuples, Ramsey's theorem becomes the infinite pigeonhole principle, which was shown by Hirst [11] to be equivalent to $B \Sigma_{2}^{0}$ over $R C A_{0}$. Ramsey's theorem for pairs is a more complicated case. It is strictly weaker than $A C A_{0}$ (Seetpun, see [18] and [12]), and not equivalent to any of the standard second-order systems. Some recent results in the ongoing investigation of the strength of Ramsey's theorem for pairs and of related, generally weaker, combinatorial results, can be found in Cholak, Jockusch, and Slaman [1],

Received June 11, 2012; accepted July 23, 2013

First published online September 9, 2015

2010 Mathematics Subject Classification: Primary 03B30; Secondary 05C55, 03F35

Keywords: reverse mathematics, Ramsey, partitions, partial orderings

(C) 2016 by University of Notre Dame 10.1215/00294527-3314771 


\title{
Reverse Mathematics and Ramsey Properties of Partial Orderings
}

\author{
Jared Corduan and Marcia Groszek
}

\begin{abstract}
A partial ordering $\mathbb{P}$ is $n$-Ramsey if, for every coloring of $n$-element chains from $\mathbb{P}$ in finitely many colors, $\mathbb{P}$ has a homogeneous subordering isomorphic to $\mathbb{P}$. In their paper on Ramsey properties of the complete binary tree, Chubb, Hirst, and McNicholl ask about Ramsey properties of other partial orderings. They also ask whether there is some Ramsey property for pairs equivalent to $A C A_{0}$ over $R C A_{0}$.

A characterization theorem for finite-level partial orderings with Ramsey properties has been proven by the second author. We show, over $R C A_{0}$, that one direction of the equivalence given by this theorem is equivalent to $A C A_{0}$ (for $n \geq 3$ ), and the other is provable in $A T R_{0}$.

We answer Chubb, Hirst, and McNicholl's second question by showing that there is a primitive recursive partial ordering $\mathbb{P}$ such that, over $R C A_{0}$, " $\mathbb{P}$ is 2-Ramsey" is equivalent to $A C A_{0}$.
\end{abstract}

\section{Introduction}

A fruitful branch of inquiry in reverse mathematics has been combinatorics, in particular, Ramsey's theorem [17] and variants thereof.

The infinitary Ramsey theorem for colorings of $n$-tuples, for standard $n \geq 3$, is equivalent to $A C A_{0}$ over $R C A_{0}$ (Simpson [19]). For colorings of 1-tuples, Ramsey's theorem becomes the infinite pigeonhole principle, which was shown by Hirst [11] to be equivalent to $B \Sigma_{2}^{0}$ over $R C A_{0}$. Ramsey's theorem for pairs is a more complicated case. It is strictly weaker than $A C A_{0}$ (Seetpun, see [18] and [12]), and not equivalent to any of the standard second-order systems. Some recent results in the ongoing investigation of the strength of Ramsey's theorem for pairs and of related, generally weaker, combinatorial results, can be found in Cholak, Jockusch, and Slaman [1],

Received June 11, 2012; accepted July 23, 2013

First published online September 9, 2015

2010 Mathematics Subject Classification: Primary 03B30; Secondary 05C55, 03F35

Keywords: reverse mathematics, Ramsey, partitions, partial orderings

(C) 2016 by University of Notre Dame 10.1215/00294527-3314771 


\title{
Reverse Mathematics and Ramsey Properties of Partial Orderings
}

\author{
Jared Corduan and Marcia Groszek
}

\begin{abstract}
A partial ordering $\mathbb{P}$ is $n$-Ramsey if, for every coloring of $n$-element chains from $\mathbb{P}$ in finitely many colors, $\mathbb{P}$ has a homogeneous subordering isomorphic to $\mathbb{P}$. In their paper on Ramsey properties of the complete binary tree, Chubb, Hirst, and McNicholl ask about Ramsey properties of other partial orderings. They also ask whether there is some Ramsey property for pairs equivalent to $A C A_{0}$ over $R C A_{0}$.

A characterization theorem for finite-level partial orderings with Ramsey properties has been proven by the second author. We show, over $R C A_{0}$, that one direction of the equivalence given by this theorem is equivalent to $A C A_{0}$ (for $n \geq 3$ ), and the other is provable in $A T R_{0}$.

We answer Chubb, Hirst, and McNicholl's second question by showing that there is a primitive recursive partial ordering $\mathbb{P}$ such that, over $R C A_{0}$, " $\mathbb{P}$ is 2-Ramsey" is equivalent to $A C A_{0}$.
\end{abstract}

\section{Introduction}

A fruitful branch of inquiry in reverse mathematics has been combinatorics, in particular, Ramsey's theorem [17] and variants thereof.

The infinitary Ramsey theorem for colorings of $n$-tuples, for standard $n \geq 3$, is equivalent to $A C A_{0}$ over $R C A_{0}$ (Simpson [19]). For colorings of 1-tuples, Ramsey's theorem becomes the infinite pigeonhole principle, which was shown by Hirst [11] to be equivalent to $B \Sigma_{2}^{0}$ over $R C A_{0}$. Ramsey's theorem for pairs is a more complicated case. It is strictly weaker than $A C A_{0}$ (Seetpun, see [18] and [12]), and not equivalent to any of the standard second-order systems. Some recent results in the ongoing investigation of the strength of Ramsey's theorem for pairs and of related, generally weaker, combinatorial results, can be found in Cholak, Jockusch, and Slaman [1],

Received June 11, 2012; accepted July 23, 2013

First published online September 9, 2015

2010 Mathematics Subject Classification: Primary 03B30; Secondary 05C55, 03F35

Keywords: reverse mathematics, Ramsey, partitions, partial orderings

(C) 2016 by University of Notre Dame 10.1215/00294527-3314771 


\title{
Reverse Mathematics and Ramsey Properties of Partial Orderings
}

\author{
Jared Corduan and Marcia Groszek
}

\begin{abstract}
A partial ordering $\mathbb{P}$ is $n$-Ramsey if, for every coloring of $n$-element chains from $\mathbb{P}$ in finitely many colors, $\mathbb{P}$ has a homogeneous subordering isomorphic to $\mathbb{P}$. In their paper on Ramsey properties of the complete binary tree, Chubb, Hirst, and McNicholl ask about Ramsey properties of other partial orderings. They also ask whether there is some Ramsey property for pairs equivalent to $A C A_{0}$ over $R C A_{0}$.

A characterization theorem for finite-level partial orderings with Ramsey properties has been proven by the second author. We show, over $R C A_{0}$, that one direction of the equivalence given by this theorem is equivalent to $A C A_{0}$ (for $n \geq 3$ ), and the other is provable in $A T R_{0}$.

We answer Chubb, Hirst, and McNicholl's second question by showing that there is a primitive recursive partial ordering $\mathbb{P}$ such that, over $R C A_{0}$, " $\mathbb{P}$ is 2-Ramsey" is equivalent to $A C A_{0}$.
\end{abstract}

\section{Introduction}

A fruitful branch of inquiry in reverse mathematics has been combinatorics, in particular, Ramsey's theorem [17] and variants thereof.

The infinitary Ramsey theorem for colorings of $n$-tuples, for standard $n \geq 3$, is equivalent to $A C A_{0}$ over $R C A_{0}$ (Simpson [19]). For colorings of 1-tuples, Ramsey's theorem becomes the infinite pigeonhole principle, which was shown by Hirst [11] to be equivalent to $B \Sigma_{2}^{0}$ over $R C A_{0}$. Ramsey's theorem for pairs is a more complicated case. It is strictly weaker than $A C A_{0}$ (Seetpun, see [18] and [12]), and not equivalent to any of the standard second-order systems. Some recent results in the ongoing investigation of the strength of Ramsey's theorem for pairs and of related, generally weaker, combinatorial results, can be found in Cholak, Jockusch, and Slaman [1],

Received June 11, 2012; accepted July 23, 2013

First published online September 9, 2015

2010 Mathematics Subject Classification: Primary 03B30; Secondary 05C55, 03F35

Keywords: reverse mathematics, Ramsey, partitions, partial orderings

(C) 2016 by University of Notre Dame 10.1215/00294527-3314771 


\title{
Reverse Mathematics and Ramsey Properties of Partial Orderings
}

\author{
Jared Corduan and Marcia Groszek
}

\begin{abstract}
A partial ordering $\mathbb{P}$ is $n$-Ramsey if, for every coloring of $n$-element chains from $\mathbb{P}$ in finitely many colors, $\mathbb{P}$ has a homogeneous subordering isomorphic to $\mathbb{P}$. In their paper on Ramsey properties of the complete binary tree, Chubb, Hirst, and McNicholl ask about Ramsey properties of other partial orderings. They also ask whether there is some Ramsey property for pairs equivalent to $A C A_{0}$ over $R C A_{0}$.

A characterization theorem for finite-level partial orderings with Ramsey properties has been proven by the second author. We show, over $R C A_{0}$, that one direction of the equivalence given by this theorem is equivalent to $A C A_{0}$ (for $n \geq 3$ ), and the other is provable in $A T R_{0}$.

We answer Chubb, Hirst, and McNicholl's second question by showing that there is a primitive recursive partial ordering $\mathbb{P}$ such that, over $R C A_{0}$, " $\mathbb{P}$ is 2-Ramsey" is equivalent to $A C A_{0}$.
\end{abstract}

\section{Introduction}

A fruitful branch of inquiry in reverse mathematics has been combinatorics, in particular, Ramsey's theorem [17] and variants thereof.

The infinitary Ramsey theorem for colorings of $n$-tuples, for standard $n \geq 3$, is equivalent to $A C A_{0}$ over $R C A_{0}$ (Simpson [19]). For colorings of 1-tuples, Ramsey's theorem becomes the infinite pigeonhole principle, which was shown by Hirst [11] to be equivalent to $B \Sigma_{2}^{0}$ over $R C A_{0}$. Ramsey's theorem for pairs is a more complicated case. It is strictly weaker than $A C A_{0}$ (Seetpun, see [18] and [12]), and not equivalent to any of the standard second-order systems. Some recent results in the ongoing investigation of the strength of Ramsey's theorem for pairs and of related, generally weaker, combinatorial results, can be found in Cholak, Jockusch, and Slaman [1],

Received June 11, 2012; accepted July 23, 2013

First published online September 9, 2015

2010 Mathematics Subject Classification: Primary 03B30; Secondary 05C55, 03F35

Keywords: reverse mathematics, Ramsey, partitions, partial orderings

(C) 2016 by University of Notre Dame 10.1215/00294527-3314771 


\title{
Reverse Mathematics and Ramsey Properties of Partial Orderings
}

\author{
Jared Corduan and Marcia Groszek
}

\begin{abstract}
A partial ordering $\mathbb{P}$ is $n$-Ramsey if, for every coloring of $n$-element chains from $\mathbb{P}$ in finitely many colors, $\mathbb{P}$ has a homogeneous subordering isomorphic to $\mathbb{P}$. In their paper on Ramsey properties of the complete binary tree, Chubb, Hirst, and McNicholl ask about Ramsey properties of other partial orderings. They also ask whether there is some Ramsey property for pairs equivalent to $A C A_{0}$ over $R C A_{0}$.

A characterization theorem for finite-level partial orderings with Ramsey properties has been proven by the second author. We show, over $R C A_{0}$, that one direction of the equivalence given by this theorem is equivalent to $A C A_{0}$ (for $n \geq 3$ ), and the other is provable in $A T R_{0}$.

We answer Chubb, Hirst, and McNicholl's second question by showing that there is a primitive recursive partial ordering $\mathbb{P}$ such that, over $R C A_{0}$, " $\mathbb{P}$ is 2-Ramsey" is equivalent to $A C A_{0}$.
\end{abstract}

\section{Introduction}

A fruitful branch of inquiry in reverse mathematics has been combinatorics, in particular, Ramsey's theorem [17] and variants thereof.

The infinitary Ramsey theorem for colorings of $n$-tuples, for standard $n \geq 3$, is equivalent to $A C A_{0}$ over $R C A_{0}$ (Simpson [19]). For colorings of 1-tuples, Ramsey's theorem becomes the infinite pigeonhole principle, which was shown by Hirst [11] to be equivalent to $B \Sigma_{2}^{0}$ over $R C A_{0}$. Ramsey's theorem for pairs is a more complicated case. It is strictly weaker than $A C A_{0}$ (Seetpun, see [18] and [12]), and not equivalent to any of the standard second-order systems. Some recent results in the ongoing investigation of the strength of Ramsey's theorem for pairs and of related, generally weaker, combinatorial results, can be found in Cholak, Jockusch, and Slaman [1],

Received June 11, 2012; accepted July 23, 2013

First published online September 9, 2015

2010 Mathematics Subject Classification: Primary 03B30; Secondary 05C55, 03F35

Keywords: reverse mathematics, Ramsey, partitions, partial orderings

(C) 2016 by University of Notre Dame 10.1215/00294527-3314771 


\title{
Reverse Mathematics and Ramsey Properties of Partial Orderings
}

\author{
Jared Corduan and Marcia Groszek
}

\begin{abstract}
A partial ordering $\mathbb{P}$ is $n$-Ramsey if, for every coloring of $n$-element chains from $\mathbb{P}$ in finitely many colors, $\mathbb{P}$ has a homogeneous subordering isomorphic to $\mathbb{P}$. In their paper on Ramsey properties of the complete binary tree, Chubb, Hirst, and McNicholl ask about Ramsey properties of other partial orderings. They also ask whether there is some Ramsey property for pairs equivalent to $A C A_{0}$ over $R C A_{0}$.

A characterization theorem for finite-level partial orderings with Ramsey properties has been proven by the second author. We show, over $R C A_{0}$, that one direction of the equivalence given by this theorem is equivalent to $A C A_{0}$ (for $n \geq 3$ ), and the other is provable in $A T R_{0}$.

We answer Chubb, Hirst, and McNicholl's second question by showing that there is a primitive recursive partial ordering $\mathbb{P}$ such that, over $R C A_{0}$, " $\mathbb{P}$ is 2-Ramsey" is equivalent to $A C A_{0}$.
\end{abstract}

\section{Introduction}

A fruitful branch of inquiry in reverse mathematics has been combinatorics, in particular, Ramsey's theorem [17] and variants thereof.

The infinitary Ramsey theorem for colorings of $n$-tuples, for standard $n \geq 3$, is equivalent to $A C A_{0}$ over $R C A_{0}$ (Simpson [19]). For colorings of 1-tuples, Ramsey's theorem becomes the infinite pigeonhole principle, which was shown by Hirst [11] to be equivalent to $B \Sigma_{2}^{0}$ over $R C A_{0}$. Ramsey's theorem for pairs is a more complicated case. It is strictly weaker than $A C A_{0}$ (Seetpun, see [18] and [12]), and not equivalent to any of the standard second-order systems. Some recent results in the ongoing investigation of the strength of Ramsey's theorem for pairs and of related, generally weaker, combinatorial results, can be found in Cholak, Jockusch, and Slaman [1],

Received June 11, 2012; accepted July 23, 2013

First published online September 9, 2015

2010 Mathematics Subject Classification: Primary 03B30; Secondary 05C55, 03F35

Keywords: reverse mathematics, Ramsey, partitions, partial orderings

(C) 2016 by University of Notre Dame 10.1215/00294527-3314771 


\title{
Reverse Mathematics and Ramsey Properties of Partial Orderings
}

\author{
Jared Corduan and Marcia Groszek
}

\begin{abstract}
A partial ordering $\mathbb{P}$ is $n$-Ramsey if, for every coloring of $n$-element chains from $\mathbb{P}$ in finitely many colors, $\mathbb{P}$ has a homogeneous subordering isomorphic to $\mathbb{P}$. In their paper on Ramsey properties of the complete binary tree, Chubb, Hirst, and McNicholl ask about Ramsey properties of other partial orderings. They also ask whether there is some Ramsey property for pairs equivalent to $A C A_{0}$ over $R C A_{0}$.

A characterization theorem for finite-level partial orderings with Ramsey properties has been proven by the second author. We show, over $R C A_{0}$, that one direction of the equivalence given by this theorem is equivalent to $A C A_{0}$ (for $n \geq 3$ ), and the other is provable in $A T R_{0}$.

We answer Chubb, Hirst, and McNicholl's second question by showing that there is a primitive recursive partial ordering $\mathbb{P}$ such that, over $R C A_{0}$, " $\mathbb{P}$ is 2-Ramsey" is equivalent to $A C A_{0}$.
\end{abstract}

\section{Introduction}

A fruitful branch of inquiry in reverse mathematics has been combinatorics, in particular, Ramsey's theorem [17] and variants thereof.

The infinitary Ramsey theorem for colorings of $n$-tuples, for standard $n \geq 3$, is equivalent to $A C A_{0}$ over $R C A_{0}$ (Simpson [19]). For colorings of 1-tuples, Ramsey's theorem becomes the infinite pigeonhole principle, which was shown by Hirst [11] to be equivalent to $B \Sigma_{2}^{0}$ over $R C A_{0}$. Ramsey's theorem for pairs is a more complicated case. It is strictly weaker than $A C A_{0}$ (Seetpun, see [18] and [12]), and not equivalent to any of the standard second-order systems. Some recent results in the ongoing investigation of the strength of Ramsey's theorem for pairs and of related, generally weaker, combinatorial results, can be found in Cholak, Jockusch, and Slaman [1],

Received June 11, 2012; accepted July 23, 2013

First published online September 9, 2015

2010 Mathematics Subject Classification: Primary 03B30; Secondary 05C55, 03F35

Keywords: reverse mathematics, Ramsey, partitions, partial orderings

(C) 2016 by University of Notre Dame 10.1215/00294527-3314771 


\title{
Reverse Mathematics and Ramsey Properties of Partial Orderings
}

\author{
Jared Corduan and Marcia Groszek
}

\begin{abstract}
A partial ordering $\mathbb{P}$ is $n$-Ramsey if, for every coloring of $n$-element chains from $\mathbb{P}$ in finitely many colors, $\mathbb{P}$ has a homogeneous subordering isomorphic to $\mathbb{P}$. In their paper on Ramsey properties of the complete binary tree, Chubb, Hirst, and McNicholl ask about Ramsey properties of other partial orderings. They also ask whether there is some Ramsey property for pairs equivalent to $A C A_{0}$ over $R C A_{0}$.

A characterization theorem for finite-level partial orderings with Ramsey properties has been proven by the second author. We show, over $R C A_{0}$, that one direction of the equivalence given by this theorem is equivalent to $A C A_{0}$ (for $n \geq 3$ ), and the other is provable in $A T R_{0}$.

We answer Chubb, Hirst, and McNicholl's second question by showing that there is a primitive recursive partial ordering $\mathbb{P}$ such that, over $R C A_{0}$, " $\mathbb{P}$ is 2-Ramsey" is equivalent to $A C A_{0}$.
\end{abstract}

\section{Introduction}

A fruitful branch of inquiry in reverse mathematics has been combinatorics, in particular, Ramsey's theorem [17] and variants thereof.

The infinitary Ramsey theorem for colorings of $n$-tuples, for standard $n \geq 3$, is equivalent to $A C A_{0}$ over $R C A_{0}$ (Simpson [19]). For colorings of 1-tuples, Ramsey's theorem becomes the infinite pigeonhole principle, which was shown by Hirst [11] to be equivalent to $B \Sigma_{2}^{0}$ over $R C A_{0}$. Ramsey's theorem for pairs is a more complicated case. It is strictly weaker than $A C A_{0}$ (Seetpun, see [18] and [12]), and not equivalent to any of the standard second-order systems. Some recent results in the ongoing investigation of the strength of Ramsey's theorem for pairs and of related, generally weaker, combinatorial results, can be found in Cholak, Jockusch, and Slaman [1],

Received June 11, 2012; accepted July 23, 2013

First published online September 9, 2015

2010 Mathematics Subject Classification: Primary 03B30; Secondary 05C55, 03F35

Keywords: reverse mathematics, Ramsey, partitions, partial orderings

(C) 2016 by University of Notre Dame 10.1215/00294527-3314771 


\title{
Reverse Mathematics and Ramsey Properties of Partial Orderings
}

\author{
Jared Corduan and Marcia Groszek
}

\begin{abstract}
A partial ordering $\mathbb{P}$ is $n$-Ramsey if, for every coloring of $n$-element chains from $\mathbb{P}$ in finitely many colors, $\mathbb{P}$ has a homogeneous subordering isomorphic to $\mathbb{P}$. In their paper on Ramsey properties of the complete binary tree, Chubb, Hirst, and McNicholl ask about Ramsey properties of other partial orderings. They also ask whether there is some Ramsey property for pairs equivalent to $A C A_{0}$ over $R C A_{0}$.

A characterization theorem for finite-level partial orderings with Ramsey properties has been proven by the second author. We show, over $R C A_{0}$, that one direction of the equivalence given by this theorem is equivalent to $A C A_{0}$ (for $n \geq 3$ ), and the other is provable in $A T R_{0}$.

We answer Chubb, Hirst, and McNicholl's second question by showing that there is a primitive recursive partial ordering $\mathbb{P}$ such that, over $R C A_{0}$, " $\mathbb{P}$ is 2-Ramsey" is equivalent to $A C A_{0}$.
\end{abstract}

\section{Introduction}

A fruitful branch of inquiry in reverse mathematics has been combinatorics, in particular, Ramsey's theorem [17] and variants thereof.

The infinitary Ramsey theorem for colorings of $n$-tuples, for standard $n \geq 3$, is equivalent to $A C A_{0}$ over $R C A_{0}$ (Simpson [19]). For colorings of 1-tuples, Ramsey's theorem becomes the infinite pigeonhole principle, which was shown by Hirst [11] to be equivalent to $B \Sigma_{2}^{0}$ over $R C A_{0}$. Ramsey's theorem for pairs is a more complicated case. It is strictly weaker than $A C A_{0}$ (Seetpun, see [18] and [12]), and not equivalent to any of the standard second-order systems. Some recent results in the ongoing investigation of the strength of Ramsey's theorem for pairs and of related, generally weaker, combinatorial results, can be found in Cholak, Jockusch, and Slaman [1],

Received June 11, 2012; accepted July 23, 2013

First published online September 9, 2015

2010 Mathematics Subject Classification: Primary 03B30; Secondary 05C55, 03F35

Keywords: reverse mathematics, Ramsey, partitions, partial orderings

(C) 2016 by University of Notre Dame 10.1215/00294527-3314771 


\title{
Reverse Mathematics and Ramsey Properties of Partial Orderings
}

\author{
Jared Corduan and Marcia Groszek
}

\begin{abstract}
A partial ordering $\mathbb{P}$ is $n$-Ramsey if, for every coloring of $n$-element chains from $\mathbb{P}$ in finitely many colors, $\mathbb{P}$ has a homogeneous subordering isomorphic to $\mathbb{P}$. In their paper on Ramsey properties of the complete binary tree, Chubb, Hirst, and McNicholl ask about Ramsey properties of other partial orderings. They also ask whether there is some Ramsey property for pairs equivalent to $A C A_{0}$ over $R C A_{0}$.

A characterization theorem for finite-level partial orderings with Ramsey properties has been proven by the second author. We show, over $R C A_{0}$, that one direction of the equivalence given by this theorem is equivalent to $A C A_{0}$ (for $n \geq 3$ ), and the other is provable in $A T R_{0}$.

We answer Chubb, Hirst, and McNicholl's second question by showing that there is a primitive recursive partial ordering $\mathbb{P}$ such that, over $R C A_{0}$, " $\mathbb{P}$ is 2-Ramsey" is equivalent to $A C A_{0}$.
\end{abstract}

\section{Introduction}

A fruitful branch of inquiry in reverse mathematics has been combinatorics, in particular, Ramsey's theorem [17] and variants thereof.

The infinitary Ramsey theorem for colorings of $n$-tuples, for standard $n \geq 3$, is equivalent to $A C A_{0}$ over $R C A_{0}$ (Simpson [19]). For colorings of 1-tuples, Ramsey's theorem becomes the infinite pigeonhole principle, which was shown by Hirst [11] to be equivalent to $B \Sigma_{2}^{0}$ over $R C A_{0}$. Ramsey's theorem for pairs is a more complicated case. It is strictly weaker than $A C A_{0}$ (Seetpun, see [18] and [12]), and not equivalent to any of the standard second-order systems. Some recent results in the ongoing investigation of the strength of Ramsey's theorem for pairs and of related, generally weaker, combinatorial results, can be found in Cholak, Jockusch, and Slaman [1],

Received June 11, 2012; accepted July 23, 2013

First published online September 9, 2015

2010 Mathematics Subject Classification: Primary 03B30; Secondary 05C55, 03F35

Keywords: reverse mathematics, Ramsey, partitions, partial orderings

(C) 2016 by University of Notre Dame 10.1215/00294527-3314771 


\title{
Reverse Mathematics and Ramsey Properties of Partial Orderings
}

\author{
Jared Corduan and Marcia Groszek
}

\begin{abstract}
A partial ordering $\mathbb{P}$ is $n$-Ramsey if, for every coloring of $n$-element chains from $\mathbb{P}$ in finitely many colors, $\mathbb{P}$ has a homogeneous subordering isomorphic to $\mathbb{P}$. In their paper on Ramsey properties of the complete binary tree, Chubb, Hirst, and McNicholl ask about Ramsey properties of other partial orderings. They also ask whether there is some Ramsey property for pairs equivalent to $A C A_{0}$ over $R C A_{0}$.

A characterization theorem for finite-level partial orderings with Ramsey properties has been proven by the second author. We show, over $R C A_{0}$, that one direction of the equivalence given by this theorem is equivalent to $A C A_{0}$ (for $n \geq 3$ ), and the other is provable in $A T R_{0}$.

We answer Chubb, Hirst, and McNicholl's second question by showing that there is a primitive recursive partial ordering $\mathbb{P}$ such that, over $R C A_{0}$, " $\mathbb{P}$ is 2-Ramsey" is equivalent to $A C A_{0}$.
\end{abstract}

\section{Introduction}

A fruitful branch of inquiry in reverse mathematics has been combinatorics, in particular, Ramsey's theorem [17] and variants thereof.

The infinitary Ramsey theorem for colorings of $n$-tuples, for standard $n \geq 3$, is equivalent to $A C A_{0}$ over $R C A_{0}$ (Simpson [19]). For colorings of 1-tuples, Ramsey's theorem becomes the infinite pigeonhole principle, which was shown by Hirst [11] to be equivalent to $B \Sigma_{2}^{0}$ over $R C A_{0}$. Ramsey's theorem for pairs is a more complicated case. It is strictly weaker than $A C A_{0}$ (Seetpun, see [18] and [12]), and not equivalent to any of the standard second-order systems. Some recent results in the ongoing investigation of the strength of Ramsey's theorem for pairs and of related, generally weaker, combinatorial results, can be found in Cholak, Jockusch, and Slaman [1],

Received June 11, 2012; accepted July 23, 2013

First published online September 9, 2015

2010 Mathematics Subject Classification: Primary 03B30; Secondary 05C55, 03F35

Keywords: reverse mathematics, Ramsey, partitions, partial orderings

(C) 2016 by University of Notre Dame 10.1215/00294527-3314771 


\title{
Reverse Mathematics and Ramsey Properties of Partial Orderings
}

\author{
Jared Corduan and Marcia Groszek
}

\begin{abstract}
A partial ordering $\mathbb{P}$ is $n$-Ramsey if, for every coloring of $n$-element chains from $\mathbb{P}$ in finitely many colors, $\mathbb{P}$ has a homogeneous subordering isomorphic to $\mathbb{P}$. In their paper on Ramsey properties of the complete binary tree, Chubb, Hirst, and McNicholl ask about Ramsey properties of other partial orderings. They also ask whether there is some Ramsey property for pairs equivalent to $A C A_{0}$ over $R C A_{0}$.

A characterization theorem for finite-level partial orderings with Ramsey properties has been proven by the second author. We show, over $R C A_{0}$, that one direction of the equivalence given by this theorem is equivalent to $A C A_{0}$ (for $n \geq 3$ ), and the other is provable in $A T R_{0}$.

We answer Chubb, Hirst, and McNicholl's second question by showing that there is a primitive recursive partial ordering $\mathbb{P}$ such that, over $R C A_{0}$, " $\mathbb{P}$ is 2-Ramsey" is equivalent to $A C A_{0}$.
\end{abstract}

\section{Introduction}

A fruitful branch of inquiry in reverse mathematics has been combinatorics, in particular, Ramsey's theorem [17] and variants thereof.

The infinitary Ramsey theorem for colorings of $n$-tuples, for standard $n \geq 3$, is equivalent to $A C A_{0}$ over $R C A_{0}$ (Simpson [19]). For colorings of 1-tuples, Ramsey's theorem becomes the infinite pigeonhole principle, which was shown by Hirst [11] to be equivalent to $B \Sigma_{2}^{0}$ over $R C A_{0}$. Ramsey's theorem for pairs is a more complicated case. It is strictly weaker than $A C A_{0}$ (Seetpun, see [18] and [12]), and not equivalent to any of the standard second-order systems. Some recent results in the ongoing investigation of the strength of Ramsey's theorem for pairs and of related, generally weaker, combinatorial results, can be found in Cholak, Jockusch, and Slaman [1],

Received June 11, 2012; accepted July 23, 2013

First published online September 9, 2015

2010 Mathematics Subject Classification: Primary 03B30; Secondary 05C55, 03F35

Keywords: reverse mathematics, Ramsey, partitions, partial orderings

(C) 2016 by University of Notre Dame 10.1215/00294527-3314771 


\title{
Reverse Mathematics and Ramsey Properties of Partial Orderings
}

\author{
Jared Corduan and Marcia Groszek
}

\begin{abstract}
A partial ordering $\mathbb{P}$ is $n$-Ramsey if, for every coloring of $n$-element chains from $\mathbb{P}$ in finitely many colors, $\mathbb{P}$ has a homogeneous subordering isomorphic to $\mathbb{P}$. In their paper on Ramsey properties of the complete binary tree, Chubb, Hirst, and McNicholl ask about Ramsey properties of other partial orderings. They also ask whether there is some Ramsey property for pairs equivalent to $A C A_{0}$ over $R C A_{0}$.

A characterization theorem for finite-level partial orderings with Ramsey properties has been proven by the second author. We show, over $R C A_{0}$, that one direction of the equivalence given by this theorem is equivalent to $A C A_{0}$ (for $n \geq 3$ ), and the other is provable in $A T R_{0}$.

We answer Chubb, Hirst, and McNicholl's second question by showing that there is a primitive recursive partial ordering $\mathbb{P}$ such that, over $R C A_{0}$, " $\mathbb{P}$ is 2-Ramsey" is equivalent to $A C A_{0}$.
\end{abstract}

\section{Introduction}

A fruitful branch of inquiry in reverse mathematics has been combinatorics, in particular, Ramsey's theorem [17] and variants thereof.

The infinitary Ramsey theorem for colorings of $n$-tuples, for standard $n \geq 3$, is equivalent to $A C A_{0}$ over $R C A_{0}$ (Simpson [19]). For colorings of 1-tuples, Ramsey's theorem becomes the infinite pigeonhole principle, which was shown by Hirst [11] to be equivalent to $B \Sigma_{2}^{0}$ over $R C A_{0}$. Ramsey's theorem for pairs is a more complicated case. It is strictly weaker than $A C A_{0}$ (Seetpun, see [18] and [12]), and not equivalent to any of the standard second-order systems. Some recent results in the ongoing investigation of the strength of Ramsey's theorem for pairs and of related, generally weaker, combinatorial results, can be found in Cholak, Jockusch, and Slaman [1],

Received June 11, 2012; accepted July 23, 2013

First published online September 9, 2015

2010 Mathematics Subject Classification: Primary 03B30; Secondary 05C55, 03F35

Keywords: reverse mathematics, Ramsey, partitions, partial orderings

(C) 2016 by University of Notre Dame 10.1215/00294527-3314771 


\title{
Reverse Mathematics and Ramsey Properties of Partial Orderings
}

\author{
Jared Corduan and Marcia Groszek
}

\begin{abstract}
A partial ordering $\mathbb{P}$ is $n$-Ramsey if, for every coloring of $n$-element chains from $\mathbb{P}$ in finitely many colors, $\mathbb{P}$ has a homogeneous subordering isomorphic to $\mathbb{P}$. In their paper on Ramsey properties of the complete binary tree, Chubb, Hirst, and McNicholl ask about Ramsey properties of other partial orderings. They also ask whether there is some Ramsey property for pairs equivalent to $A C A_{0}$ over $R C A_{0}$.

A characterization theorem for finite-level partial orderings with Ramsey properties has been proven by the second author. We show, over $R C A_{0}$, that one direction of the equivalence given by this theorem is equivalent to $A C A_{0}$ (for $n \geq 3$ ), and the other is provable in $A T R_{0}$.

We answer Chubb, Hirst, and McNicholl's second question by showing that there is a primitive recursive partial ordering $\mathbb{P}$ such that, over $R C A_{0}$, " $\mathbb{P}$ is 2-Ramsey" is equivalent to $A C A_{0}$.
\end{abstract}

\section{Introduction}

A fruitful branch of inquiry in reverse mathematics has been combinatorics, in particular, Ramsey's theorem [17] and variants thereof.

The infinitary Ramsey theorem for colorings of $n$-tuples, for standard $n \geq 3$, is equivalent to $A C A_{0}$ over $R C A_{0}$ (Simpson [19]). For colorings of 1-tuples, Ramsey's theorem becomes the infinite pigeonhole principle, which was shown by Hirst [11] to be equivalent to $B \Sigma_{2}^{0}$ over $R C A_{0}$. Ramsey's theorem for pairs is a more complicated case. It is strictly weaker than $A C A_{0}$ (Seetpun, see [18] and [12]), and not equivalent to any of the standard second-order systems. Some recent results in the ongoing investigation of the strength of Ramsey's theorem for pairs and of related, generally weaker, combinatorial results, can be found in Cholak, Jockusch, and Slaman [1],

Received June 11, 2012; accepted July 23, 2013

First published online September 9, 2015

2010 Mathematics Subject Classification: Primary 03B30; Secondary 05C55, 03F35

Keywords: reverse mathematics, Ramsey, partitions, partial orderings

(C) 2016 by University of Notre Dame 10.1215/00294527-3314771 


\title{
Reverse Mathematics and Ramsey Properties of Partial Orderings
}

\author{
Jared Corduan and Marcia Groszek
}

\begin{abstract}
A partial ordering $\mathbb{P}$ is $n$-Ramsey if, for every coloring of $n$-element chains from $\mathbb{P}$ in finitely many colors, $\mathbb{P}$ has a homogeneous subordering isomorphic to $\mathbb{P}$. In their paper on Ramsey properties of the complete binary tree, Chubb, Hirst, and McNicholl ask about Ramsey properties of other partial orderings. They also ask whether there is some Ramsey property for pairs equivalent to $A C A_{0}$ over $R C A_{0}$.

A characterization theorem for finite-level partial orderings with Ramsey properties has been proven by the second author. We show, over $R C A_{0}$, that one direction of the equivalence given by this theorem is equivalent to $A C A_{0}$ (for $n \geq 3$ ), and the other is provable in $A T R_{0}$.

We answer Chubb, Hirst, and McNicholl's second question by showing that there is a primitive recursive partial ordering $\mathbb{P}$ such that, over $R C A_{0}$, " $\mathbb{P}$ is 2-Ramsey" is equivalent to $A C A_{0}$.
\end{abstract}

\section{Introduction}

A fruitful branch of inquiry in reverse mathematics has been combinatorics, in particular, Ramsey's theorem [17] and variants thereof.

The infinitary Ramsey theorem for colorings of $n$-tuples, for standard $n \geq 3$, is equivalent to $A C A_{0}$ over $R C A_{0}$ (Simpson [19]). For colorings of 1-tuples, Ramsey's theorem becomes the infinite pigeonhole principle, which was shown by Hirst [11] to be equivalent to $B \Sigma_{2}^{0}$ over $R C A_{0}$. Ramsey's theorem for pairs is a more complicated case. It is strictly weaker than $A C A_{0}$ (Seetpun, see [18] and [12]), and not equivalent to any of the standard second-order systems. Some recent results in the ongoing investigation of the strength of Ramsey's theorem for pairs and of related, generally weaker, combinatorial results, can be found in Cholak, Jockusch, and Slaman [1],

Received June 11, 2012; accepted July 23, 2013

First published online September 9, 2015

2010 Mathematics Subject Classification: Primary 03B30; Secondary 05C55, 03F35

Keywords: reverse mathematics, Ramsey, partitions, partial orderings

(C) 2016 by University of Notre Dame 10.1215/00294527-3314771 


\title{
Reverse Mathematics and Ramsey Properties of Partial Orderings
}

\author{
Jared Corduan and Marcia Groszek
}

\begin{abstract}
A partial ordering $\mathbb{P}$ is $n$-Ramsey if, for every coloring of $n$-element chains from $\mathbb{P}$ in finitely many colors, $\mathbb{P}$ has a homogeneous subordering isomorphic to $\mathbb{P}$. In their paper on Ramsey properties of the complete binary tree, Chubb, Hirst, and McNicholl ask about Ramsey properties of other partial orderings. They also ask whether there is some Ramsey property for pairs equivalent to $A C A_{0}$ over $R C A_{0}$.

A characterization theorem for finite-level partial orderings with Ramsey properties has been proven by the second author. We show, over $R C A_{0}$, that one direction of the equivalence given by this theorem is equivalent to $A C A_{0}$ (for $n \geq 3$ ), and the other is provable in $A T R_{0}$.

We answer Chubb, Hirst, and McNicholl's second question by showing that there is a primitive recursive partial ordering $\mathbb{P}$ such that, over $R C A_{0}$, " $\mathbb{P}$ is 2-Ramsey" is equivalent to $A C A_{0}$.
\end{abstract}

\section{Introduction}

A fruitful branch of inquiry in reverse mathematics has been combinatorics, in particular, Ramsey's theorem [17] and variants thereof.

The infinitary Ramsey theorem for colorings of $n$-tuples, for standard $n \geq 3$, is equivalent to $A C A_{0}$ over $R C A_{0}$ (Simpson [19]). For colorings of 1-tuples, Ramsey's theorem becomes the infinite pigeonhole principle, which was shown by Hirst [11] to be equivalent to $B \Sigma_{2}^{0}$ over $R C A_{0}$. Ramsey's theorem for pairs is a more complicated case. It is strictly weaker than $A C A_{0}$ (Seetpun, see [18] and [12]), and not equivalent to any of the standard second-order systems. Some recent results in the ongoing investigation of the strength of Ramsey's theorem for pairs and of related, generally weaker, combinatorial results, can be found in Cholak, Jockusch, and Slaman [1],

Received June 11, 2012; accepted July 23, 2013

First published online September 9, 2015

2010 Mathematics Subject Classification: Primary 03B30; Secondary 05C55, 03F35

Keywords: reverse mathematics, Ramsey, partitions, partial orderings

(C) 2016 by University of Notre Dame 10.1215/00294527-3314771 


\title{
Reverse Mathematics and Ramsey Properties of Partial Orderings
}

\author{
Jared Corduan and Marcia Groszek
}

\begin{abstract}
A partial ordering $\mathbb{P}$ is $n$-Ramsey if, for every coloring of $n$-element chains from $\mathbb{P}$ in finitely many colors, $\mathbb{P}$ has a homogeneous subordering isomorphic to $\mathbb{P}$. In their paper on Ramsey properties of the complete binary tree, Chubb, Hirst, and McNicholl ask about Ramsey properties of other partial orderings. They also ask whether there is some Ramsey property for pairs equivalent to $A C A_{0}$ over $R C A_{0}$.

A characterization theorem for finite-level partial orderings with Ramsey properties has been proven by the second author. We show, over $R C A_{0}$, that one direction of the equivalence given by this theorem is equivalent to $A C A_{0}$ (for $n \geq 3$ ), and the other is provable in $A T R_{0}$.

We answer Chubb, Hirst, and McNicholl's second question by showing that there is a primitive recursive partial ordering $\mathbb{P}$ such that, over $R C A_{0}$, " $\mathbb{P}$ is 2-Ramsey" is equivalent to $A C A_{0}$.
\end{abstract}

\section{Introduction}

A fruitful branch of inquiry in reverse mathematics has been combinatorics, in particular, Ramsey's theorem [17] and variants thereof.

The infinitary Ramsey theorem for colorings of $n$-tuples, for standard $n \geq 3$, is equivalent to $A C A_{0}$ over $R C A_{0}$ (Simpson [19]). For colorings of 1-tuples, Ramsey's theorem becomes the infinite pigeonhole principle, which was shown by Hirst [11] to be equivalent to $B \Sigma_{2}^{0}$ over $R C A_{0}$. Ramsey's theorem for pairs is a more complicated case. It is strictly weaker than $A C A_{0}$ (Seetpun, see [18] and [12]), and not equivalent to any of the standard second-order systems. Some recent results in the ongoing investigation of the strength of Ramsey's theorem for pairs and of related, generally weaker, combinatorial results, can be found in Cholak, Jockusch, and Slaman [1],

Received June 11, 2012; accepted July 23, 2013

First published online September 9, 2015

2010 Mathematics Subject Classification: Primary 03B30; Secondary 05C55, 03F35

Keywords: reverse mathematics, Ramsey, partitions, partial orderings

(C) 2016 by University of Notre Dame 10.1215/00294527-3314771 